\title{
Mobile Banking Adeptness on Man-In-The-Middle and Man-In-The-Browser Attacks
}

\author{
P.S.Jagadeesh Kumar, Wenli Hu, Xianpei Li, Kundan Lal
}

\begin{abstract}
A brand new category of perilous threat, asphyxiate the browser behavior in line of attack similar to that of Trojan horses. These immaculate varieties of Trojan horses can acclimatize the banking transaction activities, as they are muddled in browsers on top of mobile banking applications, and exhibit the user's transactions. Primarily, they are man-in-the-middle (MITM) attacks pedestal on users illuminating their testament on a deceptive website and man-in-the-browser (MITB) attacks that modify the exterior of indenture in the user's browser. Repugnant to phishing attacks which depends upon similar but illusive websites, these malicious attacks cannot be professed by the user, as they are with genuine titivates, the user is desperately logged-in as standard, and consequently, falls prey in losing practical mobile banking utilities.
\end{abstract}

Keywords: Man-In-The-Middle (MITM), Man-In-The-Browser (MITB), Mobile Banking, Phishing Attack

\section{Introduction}

Mobile conventional password based consumer substantiation has been a distinguished sanctuary predicament. Even a primordial and tenuously accomplished phishing attack can be effectual for an effortless password thievery. Attributable to accumulate of phishing and connected attacks, susceptible requests such as online banking gradually more desired for more safe and sound authentication substitutes. Their clarifications, typically drop into a collection called two-step verification. By having a derived step, consumer authentication not only strives on something known but as well on a little you have. Therefore, positioning a false locate for gathering user codeword is no longer satisfactory to demeanor a flourishing attack. There is a restrained but significant dissimilarity involving a phishing attack and a Man-In-The-Middle (MITM) attack. Though a phishing situate is stationary and unreceptive, a MITM attack is a dynamic attack performed instantaneously [1]. Two-step verification by itself does not bequeath with fortification beside MITM attacks. One tendency in topical days that transforms the setting of authentication is the augment of mobile strategy. Previous year, it was foreseen that by the end of 2015 smart phones will overhaul computers as the majority web entree gadget internationally. By means of the mobile contrivance as the factor in two-step verification is originated to be further practical than hauling impressive things by users. However, present mobile elucidations like OTP over SMS as well as mobile signatures whittle up the disadvantage of deteriorating to tackle MITM attacks. The primary discrepancy involving mobile and fixed platforms are that a protected ingredient is by now offered in most of the mobile devices. On behalf of authentication rationales, the protected facet like SIM card can be employed which does an interfere defiant hardware that is competent of hoarding official documents like private keys and effect cryptographic actions without compromising keys.

E-commerce is an idiom for every brand of dealing in internet, or viable action that offers checks for import and exports of products or interactions of statistics crosswise the internet. A range of E-commerce relevance includes online shopping, e-tickets, banking, and so on. An online business structure is a recompense technique that permits transmit of subsidizes over an Electronic Fund Transfer (EFT). In online commerce, customers pay through their credit or debit card to procure artifacts from vendor. In many countries, populace is utilizing these examine for auction and procure. Credit and debit cards have progressively become the desired mode of recompenses for goods and services, building this outline of electronic imbursements an obligatory approach for commercials large and undersized to deportment commerce. Online utilities abridge our lives [2]. They permit us to way in data universally and are also practical for service providers since they trim down the equipped costs concerned in proposing a service. For instance, online banking utility over the internet has befallen indispensable for consumers as well as for stockpiles. Despite all these advantages, ecommerce is not fully protected and has associated risks.

\section{MITM Attack}

MITM attacks rely on clientele revealing their testimonials on an illusory website as shown in Fig.1. The assailant then pleads with the justifiable diplomas to sign onto the rightful site for example a bank entry, and then performs as a relay among the rightful user and the justifiable site. What is abnormal regarding the MITM attacks is that they thrive despite clients by means of one time password (OTP) tokens that produce an inimitable code word each time. The assailant instantly frontwards the client's diplomas to the bank gateway, marking in prior to the token engendered onetime password can perish. MITM attacks create the chore of 
maintaining data protected and confidential chiefly exigent because attacks can be mount from distant computers with counterfeit addresses. Whereas security was principally the breaking of encryption changes, the difficulty of sanctuary in computer groups also engages dynamic intrusion by interlopers, and of these MIM attack is one of the most fabulous. The MIM attack acquires benefit of the Achilles' heel in the verification etiquettes being worned by the corresponding revelry. Since authentication is usually afforded by third parties who subject credentials, the scheme of certificate making befalls one more spring of possible Achilles' heel. The MIM attack permits the interloper or the illegal revelry to get in the way on data from side to side the backdoor. This intercession is also being utilized by groups to meddle upon their workers and for adware. For example, in the early hours 2015, it was revealed that Lenovo computers came preinstalled with adware called Superfish that introduces promotion on browsers like Google Chrome and Internet Explorer. Superfish set up a self-breed root certificate into the Windows certificate hoard and then quit all SSL certificates offered by HTTPS sites with its individual credentials. This may perhaps permit hackers to potentially embezzle receptive data like banking diplomas or to scout on the users' actions. An illustration of an offline MIM attack is the interruption of an epistle by the mailman who moreover just interprets its substances or even reinstates its substances. One can envisage an online MIM attack in a civic situate like a shopping mall that affords free Wi-Fi bond accessible through a wireless router with spiteful software installed. If a consumer stopovers a bank's website at that instance from mobile phone or laptop, she might finish up trailing bank diplomas. These assails can be originated as of the subsequent rationales: ARP Cache Poisoning, DNS Spoofing, Session Hijacking, and SSL Hijacking [3].

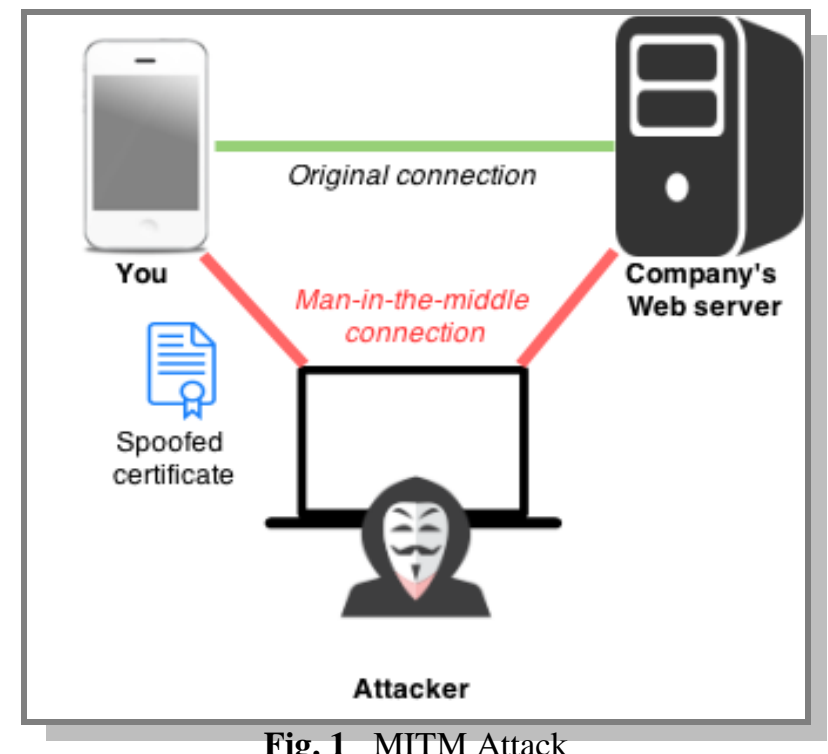

\section{A. Address Resolution Protocol (ARP) Cache Poisoning}

In the standard ARP connection, the host PC will propel a packet which has the source and destination IP address within the packet and will transmit it to all the machines associated to the network. The machine which has the intention IP address will only propel the ARP respond with its MAC address in it and then the connection is established. The ARP protocol is not a tenable protocol and the ARP cache do not have an infallible method which effects in a large predicament. The ARP retort packet can be effortlessly spoofed and it can be flinted to the device which flings the ARP request without eloquent that this is not the definite device, but an attack to origin data infringes. This occurs since the ARP cache table will be rationalized as determined by the assailant and so all the network passage will go from side to side the assailant and she will have all the information and utilize the most out of it. This is the most excellent type of attack on the Local Area Network as shown in Fig.2. Dissimilar kinds of contrivances are accessible in the bazaar for ARP Cache poisoning the occasional of those are Ettercap, Cain, Dsniff and Abel's etc. ARP Cache poisoning can be controlled by means of Dynamic ARP Inspection (DAI). DAI is a sanctuary characteristic that is exercised to authenticate the ARP packets in a network and to abandon the negated IP to MAC address requisites. The assessment should be conceded out on the Ethernet toggles by physically organizing them, but these cannot be completed on the controls which are not attuned with this examination. The ARP request and retort do not necessitate any validation or corroboration because all the swarms on the network will trust the ARP responds. The ARP cache table can be updated by interleaving a motionless admission for the ingress so the assailant would not maneuver with the gateway access in the table but this is not an infallible solution [4]. 


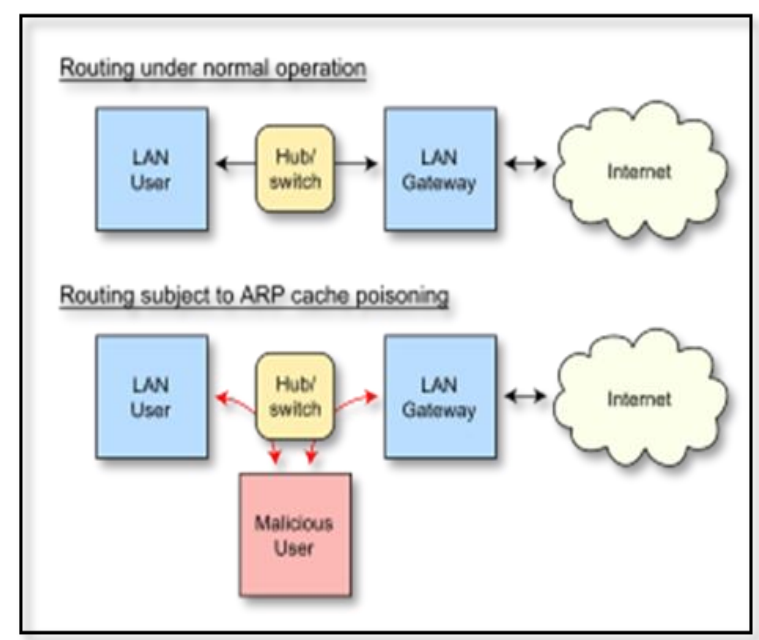

Fig. 2 ARC Cache Poisoning

\section{B. DNS Spoofing}

The intention, in this crate, will be offered with replica sequences which would guide to pasting of records. This is a sort of online MIM attack where the assailant has fashioned a counterfeit website of the bank, so while visiting the bank website will be forwarded to the website shaped by the assailant and then the assailant will expand all the records. Every time entering a website on the PC, DNS appeal is sent to the DNS Server and will receive a DNS retort memorandum in the comeback [5]. This DNS demand and retort are charted jointly with an exclusive ID number. If the assailants obtain clasps of the distinctive credentials, then by camouflaging the fatality with a crooked packet enclosing the ID number the attack can be commenced. The assailant forwards the fatality to the forged website by executing ARP cache poisoning to bypass the DNS demand significance to which the forged retort is sent. The host computer desires to attach to a website to propel a DNS inquiry to the DNS server but owing to the MIM attack the assailant will interrupt this DNS query.

\section{Session Hijacking}

As the name put forwards a session is conventional when a link sandwiched between the client and a server. Transmission Control Protocol is referred as a session because it initially creates a link, then transports the information and lastly terminates the link. This is recognized as the three-way handshake procedure which demonstrates how an appropriate session seems to be as shown in Fig.3.

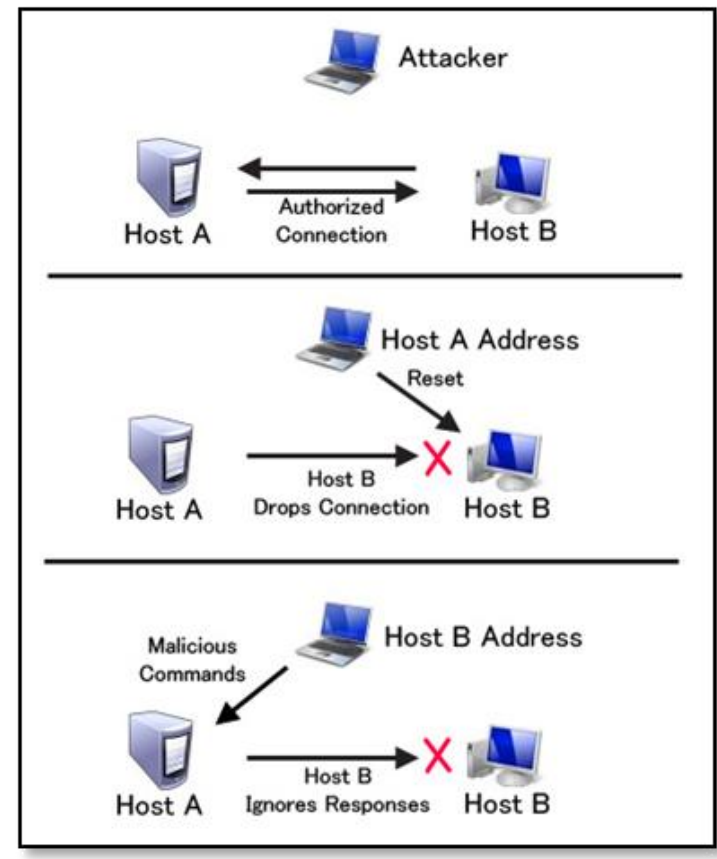

Fig. 3 Session Hijacking 
One of the renowned session hijackings is completed by pilfering cookies with the assist of Hyper Text Transfer Protocol (HTTP). In several websites one involves username and password for verification and ascertaining the session. Once the session is customary, except and waiting of the session is not completed, so to direct session cookies are utilizing the presented data that the session is still enduring. If the assailant obtains grasp of this cookie she can have the session statistics which may be enigmatic.

\section{SSL Hijacking}

Once the session is recognized amid the host PC and the web server the assailant can get hold of convinced fractions of the session connection which is completed by incarcerating the cookies that were worned for the session connection. As shown in Fig.4 following the session connection the assailant acquires the session data involving the host PC and the web server and manages the session. One desires to give security while linking with network devices can be acquired with the assist of Secure Socket Layers (SSL) or Transport Layer Security (TLS) by means of encryption tactics. This procedure is employed with other etiquettes for protected execution of the utilities that the protocol affords. HTTPS is the frequently exploited practice and much of the online banking utilities and email services employ it to guarantee sanctuary connecting their servers and the web browser [6].

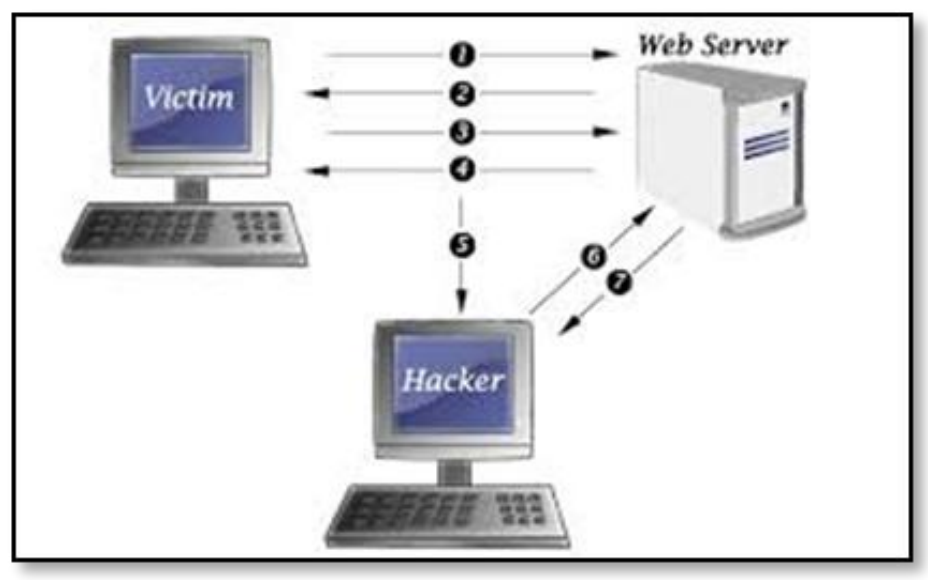

Fig. 4 Preventing SSL Hijacking

\section{MITB Attack}

The MITB threat exploits a malware Trojan on a fatality's system that is proficient to adapt Web transactions as they transpire in real time as shown in Fig. 5. The Trojan does not arbitrate until after a consumer has corroborated himself with his monetary institution by means of any authentication tools, together with OTP tokens, smartcards and PKI. Once associated to the justifiable site and 'take credit' on a lawful authentic session amid the consumer and the monetary institution, the MITB attack changes the exterior of contracts in the consumer's browser. As the modification happens in real-time, the MITB avoids the consumer from perceiving the untrustworthy commotion. This attack is hazardous since its propensity to incomprehensible from anti-virus software and pilfers information a purchaser types into the browser.

MITB is proficient to observe data contained by the browser. Because no encryption happens inside the browser, safety controls employed by pecuniary societies are ineffective. Two-step verification might also be futile if the malware has access to consumer account settings [7]. Anti-fraud methods that banks employ to perceive malicious fuss are unproductive since the transactions happen from the customer's terminal. Many banks have enhanced extra layers of safety for wire transfers by means of warnings such as SMS texts. Although, if an assailant is clever to embezzle consumers records then an assailant may have the aptitude to modify notification locales in the customer's bank account.

With respect to MITB attacks, many network echelon machines like web request firewalls, IDS and IPS schemes have complexity in sensing this attack as it happens locally on the customer side. Decrypting SSL banking sessions might be a resolution, but might generate a repercussion from consumers and executive who necessitate solitude. What formulates MITB attacks popular is the simplicity to which it can be organized to numerous methods at once through phishing links or by negotiating justifiable sites. By snapping a link, Trojan malware can be established with superfluities into a browser that has not been correctly protected. More assailants are stirring away from the conventional MITM attack to the MITB attack for such grounds. The disparity amid MITB and MITM attacks is in their maneuver. MITM attacks employ a proxy flanked by two methods that execute a transaction. 


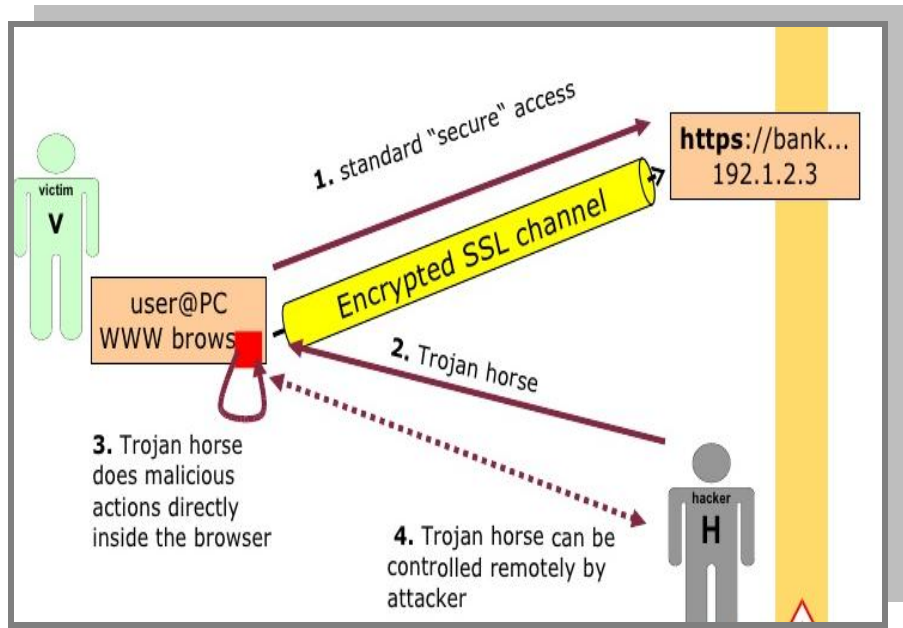

Fig. 5 MITB Attack

\section{A. Browser Helper Objects (BHO)}

Browser Helper Objects are DLL (dynamic linked libraries) units which can access DOM (Document Object Model) within a browser. Browser Helper Objects were fashioned by Microsoft and sprint in the address space of the browser and implant the main window of the browser. They are established as accompaniments to the browser for additional utility. The concern with Browser Helper Objects is their capacity to scuttle with system echelon dispensations on the operating system. Browser Helper Objects have been a familiar technique for hackers to the exploitation due to their capability to conceal from anti-virus software. MITB attacks can utilize browser helper objects to alter a site, accumulating fields or eliminating fields as an instance. Browser helper objects can still append registry entry to the method, which will stack at set up when a browser is opened. Add-ons have been recognized to employ MITB attacks, such as JavaScript and ActiveX controls to manage the browser. One add-on that is popular with Firefox is Grease Monkey. Grease Monkey (Monkey-in-the-Browser) for Firefox and Tamper Monkey for Chrome pertain the similar tactic to a MITB attack in that their utility is to modify what is sighted when breaking websites, such as purging ads from the monitor or altering the manifestation of a website. There characteristics are to develop the customers practice relatively than filch data, but the line of attack is identical. This is prepared with consumer scripts, which are JavaScript applets that can be shared within the society. Customer scripts utilized within add-ons are much more influential than customary JavaScript programs, since they can maneuver and regain confidential data in a customer's browser exclusive of Same-Origin Policy (SOP) restrictions. Malware such as Zeus that make use of MITB traits use configuration files to renew scripts for the browser to bring into play [8].

\section{B. DOM Module Interface}

The major technique for MITB to effort is by the DOM Module Interface. The rapidity that happens throughout this procedure is as pursues. Once the Trojan is established it will put in a conservatory into the browser design. This will root the conservatory to refill when the browser begins back up. When the extension is loaded, it schedules a supervisor for each page load. So, every time a page is loaded, the URL of the page is hunted by the extension alongside a record of recognized sites. Once the handler object notices a page it is loaded from the record and it catalogs an event button handler. Then once a page is presented, the extension extorts all data from the form fields by the DOM interface in the browser, and memorizes the significances. The conservatory then informs the browser to carry on submitting the form to the server. The server obtains the customized values in the form as a usual request, which the server cannot distinguish amid the unique value and the customized values. The server achieves the transaction and produces a delivery. The browser also obtains a receipt of the transaction. The conservatory then perceives the delivery of the URL examines the HTML for the delivery fields and reinstates the customized data in the acceptance with the unique data that was memorized in the HTML. The customer then believes that the unique transaction was received by the server unharmed and certified properly. The server attains the operation and generates deliverance.

\section{API Hooking}

MITB attacks use API Hooking to taint the browser. Once MITB is triggered from the malware, it wills effort to catch the Internet Connect function in Wininet.dll. This permits the assailant to adapt what a customer perceives in the browser. This is analogous to how HTML revising efforts. By means of methods of HTML revising the malware can modify the sites a customer browses and construct it to materialize in convinced approach still they represent the data that is not frank. Wininet, which is a superset to WinHTTP, is an API 
inside Internet Explorer that facilitates requests to interrelate with FTP and HTTP protocols to access internet resources. Many wininet utilities are beleaguered by MITB counting the httpsendrequest ( ) and navigateto ( ) functions. Some other accepted functions that are instilled comprises httpopenrequest ( ), httpsendrequest ( ), and the internetreadfile function. Amends to locales within the browser which permit this attack to be victorious will depart relics following the Registry. To shun Browser security settings that may avert a script from correctly exhibiting through a trusted site, malware may endeavor to modify security settings through the registry.

\section{Mobile Banking Utility}

User interfaces for mobile devices are inhibited by the devices' diminutive displays. Especially, mobile operating schemes and browsers require secure function identity pointers. A customer cannot definitively inform what mobile submissions or website she is interrelating with. This exposes customers to the jeopardy of gaffing a malevolent relevance for a hoped one. Mobile utilities and websites frequently connect to each other to share information or tender the customer to an associated service. For instance, a music-themed website might connect the customer to the iTunes relevance to purchase a song. In a usual utility hyperlink, the sender function connects to a subsequent object utility. After following the connection, the customer might afford the object request with confirmation diplomas or payment information. In this paper, it is conferred that phishing attacks that emulate usual inter-application links. The need of protected features displays denotes that an interapplication connection could be destabilized, and the customer would be incapable to inform that she had been propelled to the erroneous target. In a direct phishing attack, the dispatcher is a malevolent request that connects the customer to its own spoof screen instead of the actual objective application. In a MITM attack, the dispatcher is benign, but another party interrupts the link and loads a spoofed objective application in place of the proposed objective application. Phishing attacks subsist since customers befall habituated to inflowing their codeword in recognizable, frequent settings. If customers normally bump into rightful links whose objectives punctual those for confidential data, then customers will turn into trained and instinctively provide the appealing data. $70 \%$ of Smartphone users enter passwords into their phones at least once a day. A study was conducted with 250 Android applications, 250 iOS applications, and 85 websites to appraise how frequently they connect customers to password-protected or payment-related bank utilities. It was found that websites and mobile applications frequently link the customer to password-protected payment applications, thus training customers to automatically go into their diplomas after subsequent connections. Supported on this study of ordinary deeds, it was recognized that a bulky quantity of new phishing attacks against mobile platforms. It was revealed that, on Android and iOS, it is probable to construct phishing attacks that persuasively imitate the kinds of interapplication connections that the analysis originates to be ordinary. The attacks are categorized according to whether the dispatcher and objective are mobile utilities or websites [9].

MITB attacks make use of a variety of utilities and characteristics within a browser. MITB attacks occurs based on data congregated and what can be stolen comparable to form-grabbing, snapping, spamming, HTML injection and a variety of exploit utilities. This provides the assailants data on when to employ MITB as an element of the malware attack. Browser extensions are browser traits that can be second-hand to develop the operating system known the concession precise to conservatories. Browser conservatories are classically exercised to improve customer's knowledge within the browser and while browsing the Internet. Browser extensions can comprise plugins, Browser Helper Objects (BHO), JavaScript and add-on features. Many kinds of malware have been identified to employ these characteristics as fraction of a MITB attack; these comprise Zeus, URLZone, Shylock, Spyeye, Carberp and Sunspot to name a few [10].

\section{Phishing Dexterity}

Phishing is a structure of electronic identity theft in which a mixture of communal trade and website spoofing methods are exercised to hoax a customer into illuminating private data with fiscal value. In an archetypal attack, the assailant drives a large figure of spoofed electronic mails to haphazard internet customers that materialize to be impending from a rightful commercial institute such as a bank. The e-mail insists on the beneficiary to inform his personal data using links in the mail, if the beneficiary does not do so it will upshot in the suspend of his online banking description. Such un-beached threats are widespread in communal engineering attacks and are a useful practice in influencing customers. When the gullible victim trails the phishing, link offered in the electronic mail, he is aimed at a website that is beneath the influence of the assailant. The site is equipped in such a manner that it appears like the fatality by emulating the visual communal characteristics of the objective association by means of similar logos, badges and insignia.

Numerous resolutions have been urbanized to brawl against phishing attacks. Up till then, the propensity of phishing is escalating and several novel methods are employed to ground further impairment, it has thus befallen as a solemn cybercriminal commotion. Much of the cybercrimes are sourced as of conventional, fixed password which is frequently only distorted when essential: moreover, when it has expired 
or when the customer has overlooked it and desires to rearrange it. As codewords are cached on computer hard drives and amassed on servers, they are inclined to "crack". This is particularly an apprehension for people admitting their bank accounts from dissimilar positions and sometimes by an insecure network. Contrasting to a fixed password, one-time password transforms every time the customer logs-in and thus can be a resolution for the above revealed difficulties.

\section{Conclusion}

MITB attacks do have a single and apparent technique to thwart beyond exhaustive supervising and deterrence on the endpoint. Endpoint supervision that rivets observes and averts the browser from creating amends to the system is one leeway to offer some protection beside this attack. Various banks have still offered software that perceives MITB type malware. However, this is the one stratum to an attack that is incessantly sprouting. Customer edification is revealed as a technique to avoid these attacks. To the right from not burdening banking online there are numerous alternatives that can be put together to minimize the menace of this attacks subsequently. A few educational focuses to reflect on holds; configuring accounts with defends counting safe warning alternatives, inspecting account stabilities frequently, and by means of protected banks to perform operations. Thwarting browser extensions and scripting can also frontier these kinds of attacks, or avoiding scripts to sprint on SSL connections. There are techniques to confine browser extensions from executing, however definite websites may not maneuver correctly and limiting browsers is complicated in the current era of multimedia. Banks have instigated to employ tradition utilities for banking on mobile devices to circumvent any browser type incursions.

Transaction proofing is also an admired technique to thwart a MITB attack. If a customer can transform these factors online, then an assailant might modify this data to an intention of their wishing without customer knowledge. Multi-featured substantiation using voice and biometrics is an additional manner banks have initiated to employ further corroboration of a transactions legitimacy. Banks have started employing Behavioral Analysis in their techniques of shielding beside these attacks. The majority credit card corporations exercise this sanctuary quality to verify when latent swindle transpires in accounts at present. Identifying abnormal wire transfers to international accounts classically heave up a red flag as an exemplar of this sort of recognition. MITB attacks are not departing to vanish anytime almost immediately and will breed even more complicated. Prospectively stirring to mobile browsers as their make use of banking utilities is greater than before exploiting Man-in-the-mobile (MitMo) fashioned attacks.

\section{References}

[1] Kemal Bicakci, Devrim Unal, Nadir Ascioglu, Oktay Adalier, "Mobile Authentication Secure Against Man-In-The-Middle Attacks," 11th International Conference on Mobile Systems and Pervasive Computing, Procedia Computer Science 34, pp.323 329, Elsevier 2014.

[2] P.S.Jagadeesh Kumar, S.Meenakshi Sundaram, Ranjeet Kumar, “An Intellect Learning on E-mail Security and Fraud, Spam and Phishing," International Journal of Network Security \& Its Applications (IJNSA) Vol.7, No.5, September, pp.49-67, 2015.

[3] Timothy Dougan, Kevin Curran, "Man in the Browser Attacks," International Journal of Ambient Computing and Intelligence, 4(1), pp.29-39, March 2012

[4] Veelasha Moonsamy, Lynn, "Mitigating man-in-the-middle attacks on smartphones: a discussion of SSL pinning and DNSSec," $12^{\text {th }}$ Australian Information Security Management Conference, 2014.

[5] Candid Wuest, "Phishing in the Middle of the Stream - Today's Threats to Online Banking," Proceedings of the AVAR 2005 Conference.

[6] P.S.Jagadeesh Kumar, "Contemporary Isometric on Computer Network Security and Privacy," IOSR Journal of Electronics and Communication Engineering (IOSR-JECE), Volume 10, Issue 5, Oct.2015, pp.71-80.

[7] Anthony Luvanda, "Proposed Framework for Securing Mobile Banking Applications from Man in the Middle Attacks," Journal of Information Engineering and Applications, Vol.4, No.12, 2014.

[8] Nikolaos Karapanos, Srdjan Capkun, "On the Effective Prevention of TLS Man-in-the Middle Attacks in Web Applications," Proceedings of the 23rd USENIX Security Symposium, August 20-22, 2014.

[9] P.S.Jagadeesh Kumar, N.Venkateswaran, "Decorticate on Man-In-The-Middle and Man-In-The-Browser Bruising Mobile Banking Utility and Phishing Dexterity," International Journal of Computer and Information Engineering, 2016.

[10] M.Nazreen Banu, S.Munawara Banu, “A Comprehensive Study of Phishing Attacks," International Journal of Computer Science and Information Technologies, Vol. 4 (6), 2013, pp.783-786. 